T. Shioya

Nagoya Math. J.

Vol. 120 (1990), 181-204

\title{
THE IDEAL BOUNDARIES AND GLOBAL GEOMETRIC PROPERTIES OF COMPLETE OPEN SURFACES
}

\author{
TAKASHI SHIOYA
}

\section{§ 0. Introduction}

In this paper we study the ideal boundaries of surfaces admitting total curvature as a continuation of [Sy2] and [Sy3]. The ideal boundary of an Hadamard manifold is defined to be the equivalence classes of rays. This equivalence relation is the asymptotic relation of rays, defined by Busemann [Bu]. The asymptotic relation is not symmetric in general. However in Hadamard manifolds this becomes symmetric. Here it is essential that the manifolds are focal point free.

In our previous paper [Sy2] we have constructed the ideal boundary, equivalence classes of rays, of a surface admitting total curvature the Gaussian curvature of which surface may change sign. Here, if a ray $\sigma$ is asymptotic to a ray $\gamma$, then $\sigma$ and $\gamma$ are equivalent in our sense. The existence of total curvature is essential to construct our ideal boundaries. We have defined the metric on our ideal boundary, which coincides with the Tits metric due to Gromov [BGS] if the surface is Hadamard. Each connected component of the ideal boundary with the metric is either a complete 1-manifold or a single point (see [Sy2], [Sy3] and also section 1). Moreover we have proved in [Sy2] that the metric coincides with the inner distances of the geodesic circles asymptotically, and that concerns the asymptotic behavior of the Busemann functions (we review them in section 1).

Let $M$ be a finitely connected, oriented, complete and noncompact Riemannian 2-manifold without boundary. The total curvature $c(M)$ of $M$ is defined to be an improper integral $\int_{M} G d M$ of the Gaussian curvature $G$ with respect to the area element $d M$ of $M$. Throughout this paper we assume that $M$ admits total curvature. The ideal boundary $M(\infty)$ of $M$

Received November 6, 1989. 
consists of equivalence classes of rays and has the natural metric $d_{\infty}: M(\infty)$ $\times M(\infty) \rightarrow \mathbb{R} \cup\{\infty\}$ (we redefine them in section 1). We denote the class of a ray $\gamma$ by $\gamma(\infty)$. One of our results is stated as follows.

Theorem A1. For any rays $\sigma$ and $\gamma$

$$
\lim _{t \rightarrow \infty} \frac{d(\sigma(t), \gamma(t))}{t}=2 \sin \frac{\min \left\{d_{\infty}(\sigma(\infty), \gamma(\infty)), \pi\right\}}{2},
$$

where $d$ is the distance function of $M$ induced from the Riemannian metric of $M$.

Note that for any Hadamard manifold Theorem A1 holds. On an Hadamard manifold, the function $f(t):=d(\sigma(t), \gamma(t)) / t$ is monotone nondecreasing since the sectional curvatures are nonpositive everywhere. The monotonicity of $f$ concludes that (see section 4.4, [BGS])

$$
\lim _{t \rightarrow \infty} f(t) \geq 2 \sin \frac{\min \{\operatorname{Td}(\sigma(\infty), \gamma(\infty)), \pi\}}{2},
$$

where $T d$ is the Tits metric. However $f$ is not necessarily monotone in our case. Accordingly we need a delicate discussion as developed in the proof of Lemma 2.2.

For a fixed simple closed smooth curve $c$ in $M$ we set the geodesic circle by $S(t):=\{p \in M ; d(p, c)=t\}$ for $t \geq 0$. For a subset $A$ of a metric space $(X, \rho)$ we set Diam $A:=\sup \{\rho(p, q) ; p, q \in A\}$. Theorem A1 leads to the following theorem.

Theorem A2. We have

$$
\lim _{t \rightarrow \infty} \frac{\operatorname{Diam} S(t)}{t}=2 \sin \frac{\min \{\operatorname{Diam} M(\infty), \pi\}}{2} .
$$

Note that Diam $M(\infty)=(2 \pi \chi(M)-c(M)) / 2$ if $M$ has only one end, where $\chi(M)$ denotes the Euler characteristic of $M$ (see Theorem 1.5).

It is a well known fact (see section 4.7, [BGS]) that if $X$ is an Hadamard manifold, then for any $z, w \in X(\infty)$

$$
\sup _{p \in X} \Varangle_{p}(z, w)=\min \{T d(z, w), \pi\},
$$

where $\Varangle_{p}(z, w)$ is the angle at $p$ between two rays from $p$ to $z, w$. In our case we observe that this does not hold if $M$ has a bumpy metric. However we can see the asymptotic behavior of the angles as follows.

Theorem B1. Assume that $s_{i}(M) \geq 2 \pi$ for all $i$ (we define the non- 
negative value $s_{i}(M)$ for $i$-th end in section 1$)$. For any $x, y \in M(\infty)$ and for any sequence $\left\{p_{j}\right\}$ of points in $M$ such that each subsequence of $\left\{p_{j}\right\}$ diverges, let $\sigma_{j}, \gamma_{j}$ be rays emanating from $p_{j}$ such that $\sigma_{j}(\infty)=x$ and $\gamma_{j}(\infty)=y$ for all $j$. Then

$$
\limsup _{j \rightarrow \infty} \Varangle\left(\dot{\sigma}_{j}(0), \dot{\gamma}_{j}(0)\right) \leq d_{\infty}(x, y) .
$$

Note that the assumption that $s_{i}(M) \geq 2 \pi$ for all $i$ is indispensable to Theorem B1 (see Remark 3.5).

Theorem B2. For any rays $\sigma$ and $\gamma$ let $\gamma_{t}$ be a ray emanating from $\sigma(t)$ which is asymptotic to $\gamma$. Then

$$
\lim _{t \rightarrow \infty} \Varangle\left(\dot{\sigma}(t), \dot{\gamma}_{t}(0)\right)=\min \left\{d_{\infty}(\sigma(\infty), \gamma(\infty)), \pi\right\} .
$$

Here Theorem B2 holds for any Hadamard manifold (see [BGS]).

For any $x, y \in M(\infty)$ and for any subset $B$ of $M$ we set

$$
\Varangle(x, y ; B):=\sup \{\Varangle(\dot{\sigma}(0), \dot{\gamma}(0)) ; \sigma \text { and } \gamma \text { are rays emanating from }
$$

a common point in $M-B$ such that $\sigma(\infty)=x$ and $\gamma(\infty)=y$ \}.

Then Theorems B1 and B2 imply the following

Corollary B3. Assume that $s_{i}(M) \geq 2 \pi$ for all $i$. For any $x, y \in$ $M(\infty)$ and for any $p \in M$ we have

$$
\lim _{t \rightarrow \infty} \Varangle\left(x, y ; B_{t}(p)\right)=\min \left\{d_{\infty}(x, y), \pi\right\},
$$

where $B_{t}(p):=\{q \in M ; d(p, q)<t\}$.

In the final section we investigate the distribution of critical points of Busemann functions. For a Lipschitz function $f: M \rightarrow \mathbb{R}$ with Lipschitz constant 1 and for $p \in M$ we set

$$
\begin{aligned}
& V_{p}(f):=\left\{v \in T_{p} M ; \text { there exists a sequence }\left\{p_{i}\right\} \text { converging to } p\right. \\
&\text { such that } \left.f \text { is differentiable at each } p_{i} \text { and } v=\lim _{i \rightarrow \infty} \nabla f\left(p_{i}\right)\right\},
\end{aligned}
$$

where $\nabla f$ is the gradient of $f$. A point $p \in M$ is called a critical point of a Lipschitz function $f: M \rightarrow \mathbb{R}$ with Lipschitz constant 1 if for any unit vector $u \in T_{p} M$ there exists a vector $v \in V_{p}(f)$ such that $\langle u, v\rangle \geq 0$. For a ray $\gamma$ in $M$ the Busemann function $F_{r}: M \rightarrow \mathbb{R}$ is defined in [Bu] by

$$
F_{\gamma}(x):=\lim _{t \rightarrow \infty}[t-d(x, \gamma(t))] .
$$


Note that $v \in V_{p}\left(F_{r}\right)$ if and only if the geodesic $t \mapsto \exp _{p} t v$ is a ray asymptotic to $\gamma$. Here rays $\sigma$ and $\gamma$ are equivalent if $\dot{\sigma}(0) \in V_{p}\left(F_{\gamma}\right)$. We set

$\operatorname{Crit}(M):=\{p \in M ; p$ is a critical point of some Busemann function on $M\}$. Shiohama proved that if $M$ has only one end and if $2 \pi \%(M)-c(M)<\pi$, then $\operatorname{Crit}(M)$ is bounded. We extend this to the following result.

Theorem C1. If $s_{i}(M) \neq \pi$ for all $i$, then Crit $(M)$ is bounded. In particular, if $M$ has only one end and if $2 \pi \chi(M)-c(M) \neq \pi$, then Crit $(M)$ is bounded.

Note that in the case where $s_{i}(M)=\pi$ for some $i$, Crit $(M)$ is not necessarily bounded (see Remark 4.2). However we have the following

Theorem C2. If the set $\{p \in M ; G(p)=0\}$ is compact, then Crit $(M)$ is bounded.

\section{$\S 1$. Preliminaries}

In this section we construct the ideal boundary of $M$ and review the results in [Sy2] and [Sy3]. Since $M$ is finitely connected, there are a closed 2-manifold $N$ and different points $e_{1}, \cdots, e_{k} \in N$ (we call them ends) such that $M$ is homeomorphic to $N-\left\{e_{1}, \cdots, e_{k}\right\}$. Let $\varphi: M \rightarrow N-$ $\left\{e_{1}, \cdots, e_{k}\right\}$ be a homeomorphism. For each end $e_{i}$ we define a set $\mathscr{U}\left(e_{i}\right)$ of closed half cyclinders in $M$ by this condition: $U \in \mathscr{U}\left(e_{i}\right)$ if and only if the subset $\varphi(U) \cup\left\{e_{i}\right\}$ of $N$ is a closed disk and $\partial U$, the boundary of $U$, consists of a simple closed smooth curve. According to Busemann [Bu] we call an element of $\mathscr{U}\left(e_{i}\right)$ a tube of $M$. For any domain $D$ in $M$ such that $\partial D$ consists of finitely many piecewise smooth curves which are parametrized positively relative to $D$, we denote by $\kappa(D)$ the sum of integrals of geodesic curvatures of $\partial D$ and of exterior angles of $D$ at all vertices. Then the Gauss-Bonnet theorem implies $c(D)=2 \pi \chi(D)-\kappa(D)$, where $c(D):=\int_{D} G d M$. If we set $s_{i}(M):=-c(U)-\kappa(U)$ for a tube $U \in \mathscr{U}\left(e_{i}\right)$, then this is independent of the choice of $U$, and we have

$$
\sum_{i=1}^{k} s_{i}(M)=2 \pi \chi(M)-c(M)
$$

by the Gauss-Bonnet theorem. Here $0 \leq s_{i}(M) \leq+\infty$ follows from CohnVossen's results (see [Col] and also 43, [Bu]). For any ray $\gamma$ in $M$ a 
number $n(\gamma) \in\{1, \cdots, k\}$ is uniquely determined by $\lim _{t \rightarrow \infty} \varphi \circ \gamma(t)=e_{n(r)}$. It follows that for any $U \in \mathscr{U}\left(e_{n(r)}\right)$ there is a subray of $\gamma$ contained in $U$. For arbitrary given rays $\sigma_{j}$ for $j=1, \cdots, m$ with $n\left(\sigma_{j}\right)=i$ we choose a tube $U \in \mathscr{U}\left(e_{i}\right)$ in such a way that

(a) each $\sigma_{j}(0)$ is contained in $M-\operatorname{Int}(U)$, where $\operatorname{Int}(A)$ denotes the interior of a set $A$,

(b) each $\dot{\sigma}\left(t_{\sigma_{j}}\right)$ is perpendicular to $\partial U$, where $t_{\sigma_{j}}:=\sup \left\{t \geq 0 ; \sigma_{i}(t) \in \partial U\right\}$.

(c) for all different numbers $j$ and $j^{\prime}, \sigma_{j}\left(\left[t_{\sigma_{j}}, \infty\right)\right)$ does not intersect $\sigma_{j^{\prime}}\left(\left[t_{\sigma_{j^{\prime}}}, \infty\right)\right)$ otherwise $\sigma_{j}\left(\left[t_{\sigma_{j}}, \infty\right)\right)=\sigma_{j^{\prime}}\left(\left[t_{\sigma_{j^{\prime}}}, \infty\right)\right)$.

We denote by $\mathscr{U}_{\sigma_{1}, \ldots, \sigma_{m}}\left(e_{i}\right)$ the set of all tubes in $\mathscr{U}\left(e_{i}\right)$ satisfying (a), (b) and (c).

For arbitrary given rays $\sigma$ and $\gamma$ we get a tube $U \in \mathscr{U}_{\sigma, \tau}\left(e_{\imath}\right)$. By definition, $\partial U$ consists of a simple closed smooth curve $c$. We assume that $c$ is parametrized positively relative to $U$ and that $\kappa$ is the geodesic curvature of $c$. Let $I(\sigma, \gamma)$ be the closed subarc of $c$ from $\sigma\left(t_{\sigma}\right)$ to $\gamma\left(t_{\gamma}\right)$ and $D(\sigma, \gamma)$ the closed half plane in $U$ bounded by $\sigma\left(\left[t_{\sigma}, \infty\right)\right) \cup I(\sigma, \gamma) \cup \gamma\left(\left[t_{\gamma}, \infty\right)\right)$. In the special case where $\sigma\left(\left[t_{\sigma}, \infty\right)\right)=\gamma\left(\left[t_{r}, \infty\right)\right)$, we set $I(\sigma, \gamma):=\left\{\sigma\left(t_{\sigma}\right)\right\}=\left\{\gamma\left(t_{\gamma}\right)\right\}$ and $D(\sigma, \gamma):=\sigma\left(\left[t_{\sigma}, \infty\right)\right)=\gamma\left(\left[t_{r}, \infty\right)\right)$. We often identify $I(\sigma, \gamma)$ with the interval $c^{-1}(I(\sigma, \gamma))$ and set

$$
L(\sigma, \gamma):=-c(D(\sigma, \gamma))-\int_{I(\sigma, \gamma)} \kappa d s,
$$

which is independent of the choice of $U$ by the Gauss-Bonnet theorem. Here $L(\sigma, \gamma)=0$ holds if $\sigma\left(\left[t_{\sigma}, \infty\right)\right)=\gamma\left(\left[t_{r}, \infty\right)\right)$. We have the following obvious proposition.

Proposition 1.1. For any rays $\sigma, \tau$ and $\gamma$ such that $n(\sigma)=n(\tau)=$ $n(\gamma)=: i$ and for any tube $U \in \mathscr{U}_{\sigma, \tau, r}\left(e_{i}\right)$, the following (1), (2) and (3) hold.

(1) $L(\sigma, \gamma) \geq 0$.

(2) If $\sigma\left(\left[t_{\sigma}, \infty\right)\right) \neq \gamma\left(\left[t_{\gamma}, \infty\right)\right)$, then $L(\sigma, \gamma)+L(\gamma, \sigma)=s_{i}(M)$.

(3) If $\sigma\left(t_{\sigma}\right), \tau\left(t_{\tau}\right)$ and $\gamma\left(t_{\gamma}\right)$ lie on $\partial U$ in this order, then $L(\sigma, \tau)+L(\tau, \gamma)$ $=L(\sigma, \gamma)$.

Here (1) follows from Cohn-Vossen's theorem (Satz 1, [Co2]).

Two rays $\sigma$ and $\gamma$ are called equivalent if $n(\sigma)=n(\gamma)$ and

$$
\min \{L(\sigma, \gamma), L(\gamma, \sigma)\}=0 .
$$

From Proposition 1.1 (3) this is an equivalence relation. We denote the equivalence class of a ray $\gamma$ by $\gamma(\infty)$ and the set of all equivalence classes 
by $M(\infty)$.

From Proposition 1.1 (3) the value $\min \{L(\sigma, \gamma), L(\gamma, \sigma)\}$ is independent of two representative rays $\sigma, \gamma$ chosen from the classes $\sigma(\infty), \gamma(\infty)$ with $n(\sigma)=n(\gamma)$. We define the function $d_{\infty}: M(\infty) \times M(\infty) \rightarrow \mathbb{R} \cup\{\infty\}$ by

$$
d_{\infty}(\sigma(\infty), \gamma(\infty)):= \begin{cases}\min \{L(\sigma, \gamma), L(\gamma, \sigma)\} & \text { if } n(\sigma)=n(\gamma) \\ \infty & \text { if } n(\sigma) \neq n(\gamma),\end{cases}
$$

then this becomes a distance function of $M(\infty)$ (see section 1, [Sy2]). We call the metric space $\left(M(\infty), d_{\infty}\right)$ the ideal boundary of $M$. If we set $M_{i}(\infty):=\{\gamma(\infty) \in M(\infty) ; \gamma$ is a ray in $M$ with $n(\gamma)=i\} \quad$ for $i=1, \cdots, k$, then $d_{\infty}\left(M_{i}(\infty), M_{j}(\infty)\right)=\infty$ for all different numbers $i, j$ and we have the decomposition:

$$
M(\infty)=M_{1}(\infty) \cup \cdots \cup M_{k}(\infty) .
$$

For $x \in M(\infty)$, the number $n(x)$ is naturally defined and satisfies $x \in M_{n(x)}(\infty)$.

This lemma follows from Cohn-Vossen's theorem (Satz 2, [Co2]).

Lemma 1.2. Let $\alpha: \mathbb{R} \rightarrow M$ be a piecewise smooth curve bounding a closed half plane $H$ such that $\sigma(t):=\alpha(a-t)$ and $\gamma(t):=\alpha(b+t)$ for $t \geq 0$ are rays for some constants $a, b \in \mathbb{R}$. We denote by $d_{H}$ the inner distance of $H$ and assume that $d_{H}(\sigma(t), \gamma(t)) \geq 2 t-r$ for all $t \geq 0$ and for some constant $r \geq 0$. Then

$$
L(\sigma, \gamma) \geq \pi
$$

The following proposition is a direct consequence of Lemma 1.2.

Proposition 1.3. For any straight line $\gamma: \mathbb{R} \rightarrow M$ we have $d_{\infty}(\gamma(-\infty)$, $\gamma(\infty)) \geq \pi$, where $\gamma(-\infty) \in M(\infty)$ is the class containing a ray $t \mapsto \gamma(-t)$. In particular $s_{n(r)}(M) \geq 2 \pi$ if $M$ contains a straight line $\gamma$ such that $n(\gamma(-\infty))=n(\gamma(\infty))$.

The equivalence relation of rays and the ideal boundary have the following properties.

TheOREM 1.4 (5.1, [Sy2]). If a ray $\sigma$ in $M$ is asymptotic to a ray $\gamma$, then $\sigma$ and $\gamma$ are equivalent.

Theorem 1.5 (2.4 and 5.2 in [Sy2]). For each $i$, the following (1) and (2) hold.

(1) If $s_{i}(M)=0$, then $\left(M_{i}(\infty), d_{\infty}\right)$ consists of a single point. 
(2) If $0<s_{i}(M)<+\infty$, then $\left(M_{i}(\infty), d_{\infty}\right)$ is isometric to a circle with the total length $s_{i}(M)$.

To describe $\left(M_{i}(\infty), d_{\infty}\right)$ in the case where $s_{i}(M)=+\infty$, we need some notations. For a family $\left\{I_{\lambda}\right\}_{\lambda \in A}$ of closed intervals in $\mathbb{R}$ (possibly $I_{\lambda}$ is a single point or an unbounded interval) we set

$$
S\left(\left\{I_{\lambda}\right\}_{\lambda \in \Lambda}\right):=\left\{(z, \lambda) ; z \in I_{\lambda}, \lambda \in \Lambda\right\}
$$

and define the distance function $\rho$ of $\left.S\left(\left\{I_{\lambda}\right\}\right)_{\lambda \in \Lambda}\right)$ by

$$
\rho((z, \lambda),(w, \mu)):= \begin{cases}|z-w| & \text { if } \lambda=\mu \\ \infty & \text { if } \lambda \neq \mu .\end{cases}
$$

TheOREm 1.6 (A, [Sy3]). If $s_{i}(M)=+\infty$, then there exists a family $\left\{I_{\lambda}\right\}_{\lambda \in A}$ of closed intervals in $\mathbb{R}$ such that $\left(M_{i}(\infty), d_{\infty}\right)$ is isometric to $\left(S\left(\left\{I_{\lambda}\right\}_{\lambda \in \Lambda}\right), \rho\right)$.

It is an essential property that the value $L(\sigma, \gamma)$ is equal to the length of the arc $\{\tau(\infty) \in M(\infty)$; $\tau$ is a ray contained in $D(\sigma, \gamma)\}$, which joins $\sigma(\infty)$ and $\gamma(\infty)$, for a fixed tube $U \in \mathscr{U}_{\sigma, \gamma}\left(e_{i}\right)$, where $i:=n(\sigma)=n(\gamma)$.

For a fixed simple closed smooth curve $c$ let $S(t)$ be a geodesic circle defined in section 0 . Hartman [Ha] has proved that there exists a closed and measure zero subset $E$ of $[0, \infty)$ such that for any $t \in[0, \infty)-E, S(t)$ consists of simple closed piecewise smooth curves which breaks at finitely many cut points from $c$. He has called a value in $E$ an exceptional $t$ value. Moreover Shiohama [Sh4] has proved that there exists an $R>0$ such that for any $t \geq R, S(t)$ is homeomorphic to the disjoint union of $k$ circles, where $k$ is the number of ends of $M$. A ray $\gamma$ is called a ray from $c$ if $d(\gamma(t), c)=t$ for all $t \geq 0$. We modify Lemma 3.1 in [Sy2] to the following.

LemMa 1.7 (3.1, [Sy2]). For any rays $\sigma$ and $\gamma$ from $c$ with $n(\sigma)=n(\gamma)=: i$ and for any $U \in \mathscr{U}_{\sigma, r}\left(e_{i}\right)$, we have

$$
\lim _{t \rightarrow \infty} \frac{L(S(t) \cap D(\sigma, \gamma))}{t}=L(\sigma, \gamma),
$$

where $L(\alpha)$ denotes the length of a curve $\alpha$. In particular,

$$
\lim _{t \rightarrow \infty} \frac{L(S(t) \cap U)}{t}=s_{i}(M) \text { and } \lim _{t \rightarrow \infty} \frac{L(S(t))}{t}=2 \pi \chi(M)-c(M) .
$$

Lemma 1.7 implies the following theorem. 
Theorem 1.8 (5.3, [Sy2]). For any rays $\sigma$ and $\gamma$ from $c$, we have

$$
\lim _{t \rightarrow \infty} \frac{d_{t}(\sigma(t), \gamma(t))}{t}=d_{\infty}(\sigma(\infty), \gamma(\infty))
$$

where $d_{t}$ is the inner distance of $S(t)$.

Note that in Lemma 1.7 and Theorem 1.8 we assume that $t$ is always nonexceptional.

For arbitrary given rays $\sigma$ and $\gamma$ with $n(\sigma)=n(\gamma)=: i$ we get a tube $U \in \mathscr{U}_{\sigma, r}\left(e_{i}\right)$. We denote by $\hat{d}$ the inner distance of $D(\sigma, \gamma)$ induced from the Riemannian metric of $M$. A curve $\alpha:[0, l] \rightarrow D(\sigma, \gamma)$ is called a $\hat{d}$ segment if $L(\alpha)=\hat{d}(\alpha(0), \alpha(l))$. A curve $\tau:[0, \infty) \rightarrow M($ resp. $\tau: \mathbb{R} \rightarrow M)$ is called a $\hat{d}$-ray (resp. $\hat{d}$-line) if any subarc of $\tau$ is a $\hat{d}$-segment. Clearly any ray contained in $D(\sigma, \gamma)$ is a $\hat{d}$-ray. Under these definitions we have the following

LEMma 1.9 (4.1, [Sy2]). If $\hat{\gamma}_{t}$ for $t \geq t_{\sigma}$ denotes a $\hat{d}$-ray emanating from $\sigma(t)$ which is asymptotic to $\gamma$, then

$$
\lim _{t \rightarrow \infty} \Varangle\left(\dot{\sigma}(t), \dot{\hat{\gamma}}_{t}(t)\right)=\min \{L(\sigma, \gamma), \pi\} .
$$

We define the function $\hat{F}_{\gamma}: D(\sigma, \gamma) \rightarrow \mathbb{R}$ by

$$
\hat{F}_{r}(x):=\lim _{t \rightarrow \infty}[t-\hat{d}(x, \gamma(t))] \text {. }
$$

Then this and the Busemann function have the following properties.

LEMMA 1.10 (4.3, [Sy2]). For any rays $\sigma$ and $\gamma$ with $n(\sigma)=n(\gamma)$, we have

$$
\lim _{t \rightarrow \infty} \frac{\hat{F}_{r} \circ \sigma(t)}{t}=\cos \min \{L(\sigma, \gamma), \pi\} .
$$

Theorem 1.11 (5.5, [Sy2]). For any rays $\sigma$ and $\gamma$ we have

$$
\lim _{t \rightarrow \infty} \frac{F_{r} \circ \sigma(t)}{t}=\cos \min \left\{d_{\infty}(\sigma(\infty), \gamma(\infty)), \pi\right\}
$$

\section{§2. The distance between two rays}

Under the notations in section 1 we have the following lemma.

LEMma 2.1. For any rays $\sigma$ and $\gamma$ with $n(\sigma)=n(\gamma)$, we have

$$
\limsup _{t \rightarrow \infty} \frac{\hat{d}(\sigma(t), \gamma(t))}{t} \leq 2 \sin \frac{\min \{L(\sigma, \gamma), \pi\}}{2} \text {. }
$$


Proof. For each $t \geq \max \left\{t_{\sigma}, t_{\tau}\right\}$ let $\alpha_{t}$ be a $\hat{d}$-segment from $\sigma(t)$ to $\gamma(t)$ and let $D_{t}$ be a compact domain in $D(\sigma, \gamma)$ bounded by $I(\sigma, \gamma) \cup \sigma\left(\left[t_{\sigma}, t\right]\right) \cup$ $\gamma\left(\left[t_{r}, t\right]\right) \cup \alpha_{t}$. Then $\left\{D_{t}\right\}$ is a monotone increasing sequence. Here $D_{t}$ is a disk if $\alpha_{t}$ does not intersect $I(\sigma, \gamma)$.

We consider the case where $\cup D_{t} \neq D(\sigma, \gamma)$. Then $\alpha_{t}$ tends to a $\hat{d}$-line $\alpha$. The triangle inequality implies that

$$
\lim _{t \rightarrow \infty} \frac{\hat{d}(\sigma(t), \gamma(t))}{t}=2
$$

Moreover the minimizing property of $\alpha$ shows that $H:=D(\sigma, \gamma)$ satisfies the assumption of Lemma 1.2. Hence we have $L(\sigma, \gamma) \geq \pi$. The proof in this case is completed.

Next we consider the case where $\cup D_{t}=D(\sigma, \gamma)$. In this case, there exists a number $t_{0}$ such that $\alpha_{t}$ for each $t \geq t_{0}$ does not intersect $I(\sigma, \gamma)$. The first variation formula implies that

$$
\frac{d}{d t} \hat{d}(\sigma(t), \gamma(t))=\cos \theta(t)+\cos \varphi(t)
$$

for almost all $t \geq t_{0}$, where $\theta(t), \varphi(t)$ denote the inner angles of $D_{t}$ at $\sigma(t)$, $\gamma(t)$. Here we remark that $\hat{d}(\sigma(t), \gamma(t))$ is Lipschitz continuous by the triangle inequality. Hence

$$
\begin{aligned}
& \limsup _{t \rightarrow \infty} \frac{\hat{d}(\sigma(t), \gamma(t))}{t} \leq \limsup _{t \rightarrow \infty} \frac{d}{d t} \hat{d}(\sigma(t), \gamma(t)) \\
& \quad \leq \limsup _{t \rightarrow \infty}[\cos \theta(t)+\cos \varphi(t)] \\
& \quad \leq \limsup _{t \rightarrow \infty} 2 \cos \frac{\theta(t)+\varphi(t)}{2}=\limsup _{t \rightarrow \infty} 2 \sin \frac{\pi-\theta(t)-\varphi(t)}{2} .
\end{aligned}
$$

On the other hand, the Gauss-Bonnet theorem implies that

$$
c\left(D_{t}\right)=\theta(t)+\varphi(t)-\pi-\int_{I(a, \gamma)} \kappa d s
$$

for all $t \geq t_{0}$. Thereby

$$
\begin{aligned}
L(\sigma, \gamma) & =-c(D(\sigma, \gamma))-\int_{I(\sigma, r)} \kappa d s=-\lim _{t \rightarrow \infty} c\left(D_{t}\right)-\int_{I(\sigma, \gamma)} \kappa d s \\
& =\lim _{t \rightarrow \infty}[\pi-\theta(t)-\varphi(t)] .
\end{aligned}
$$

Thus

$$
\limsup _{t \rightarrow \infty} \frac{\hat{d}(\sigma(t), \gamma(t))}{t} \leq 2 \sin \frac{L(\sigma, \gamma)}{2}
$$


and $L(\sigma, \gamma) \leq \pi$. This completes the proof.

Lemma 2.2. For any rays $\sigma$ and $\gamma$ with $n(\sigma)=n(\gamma)$, we have

$$
\liminf _{t \rightarrow \infty} \frac{\hat{d}(\sigma(t), \gamma(t))}{t} \geq 2 \sin \frac{\min \{L(\sigma, \gamma), \pi\}}{2} .
$$

Proof. If $L(\sigma, \gamma)=0$, then Lemma 2.2 is obvious. Accordingly we assume that $L(\sigma, \gamma)>0$. Let $\alpha_{t}$ and $D_{t}$ be as in the proof of Lemma 2.1. Then by the above discussion, if $\cup D_{t} \neq D(\sigma, \gamma)$, then the formula of Lemma 2.2 holds.

We consider the case where $\cup D_{t}=D(\sigma, \gamma)$ holds. In this case, $L(\sigma, \gamma)$ $\leq \pi$ follows from the formula (*). By Theorems 1.5 and 1.6 and by the definition of $d_{\infty}$, there is a ray $\tau$ such that $L(\sigma, \tau)=L(\tau, \gamma)=L(\sigma, \gamma) / 2$. We will show that

$$
\liminf _{t \rightarrow \infty} \frac{\hat{d}\left(\sigma(t), m_{t}\right)}{t} \geq \sin L(\sigma, \tau),
$$

where $\alpha_{t}$ intersects $\tau$ at a unique point $m_{t}$ for large $t$.

We define for every tube $U^{\prime} \in \mathscr{U}_{\sigma, \gamma}\left(e_{i}\right)$ contained in $U$, the corresponding half plane $D^{\prime}(\sigma, \gamma)$ in $D(\sigma, \gamma)$ and the inner distance $\hat{d}^{\prime}$ of $D^{\prime}(\sigma, \gamma)$ by the same manner. Since $\left\{\alpha_{t}\right\}$ diverges in $D(\sigma, \gamma)$, we have

$$
\hat{d}\left(\sigma(t), m_{t}\right)=\hat{d}^{\prime}\left(\sigma(t), m_{t}\right)
$$

for all sufficiently large $t$. Since there is a tube $U^{\prime} \in \mathscr{U}_{\alpha, \tau, \tau}\left(e_{i}\right)$ contained in $U$, without loss of generality we may assume that $U \in \mathscr{U}_{\sigma, \tau, \gamma}\left(e_{i}\right)$.

Let $\left\{K_{j}\right\}$ be a monotone increasing sequence of closed disk domains with $\cup K_{j}=D(\sigma, \tau)$ such that each $\partial K_{j}$ consists of a piecewise smooth simple closed curve intersecting $I(\sigma, \gamma)$. We denote by $\hat{d}_{j}$ the inner distance of $\mathrm{Cl}\left(D(\sigma, \tau)-K_{j}\right)$, the closure of $D(\sigma, \tau)-K_{j}$. Let $s(t)$ be a number and $\beta_{j, t}$ a $\hat{d}_{j}$-segment from $\sigma(t)$ to $\tau(s(t))$ for large $t$ such that $L\left(\beta_{j, t}\right)=\hat{d}_{j}(\sigma(t), \tau)$. Let $E_{j, t}$ be a disk domain bounded by $I(\sigma, \tau) \cup \sigma\left(\left[t_{\sigma}, t\right]\right) \cup \tau\left(\left[t_{\tau}, s(t)\right]\right) \cup \beta_{j, t}$. We denote by $\theta_{j}(t)$ the inner angle of $E_{j, t}$ at $\sigma(t)$ (see Figure 2.2.f).

If there exists a number $j_{0}$ such that $U_{t} E_{j_{0}, t}=D(\sigma, \tau)$, then the first variation formula and the Gauss-Bonnet theorem imply that

$$
\frac{d}{d t} L\left(\beta_{j_{0}, t}\right)=\cos \theta_{j_{0}}(t)
$$

for almost all sufficiently large $t$ and 


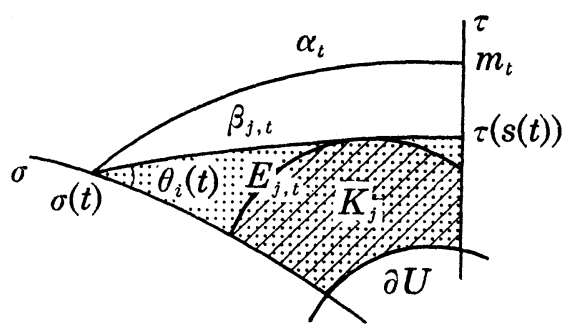

Figure 2.2.f

$$
c\left(E_{j}\right)=\theta_{j_{0}}(t)-\frac{\pi}{2}-\int_{I(\sigma, \tau)} \kappa d s
$$

for all sufficiently large $t$. Hence in this case, since $\alpha_{t}$ does not intersect $K_{j_{0}}$ for all sufficiently large $t$, we have $\hat{d}\left(\sigma(t), m_{t}\right) \geq L\left(\beta_{j_{0}, t}\right)$. Therefore

$$
\begin{aligned}
\liminf _{t \rightarrow \infty} \frac{\hat{d}\left(\sigma(t), m_{t}\right)}{t} & \geq \liminf _{t \rightarrow \infty} \frac{L\left(\beta_{j_{0}, t}\right)}{t} \geq \liminf _{t \rightarrow \infty} \frac{d}{d t} L\left(\beta_{j_{0}, t}\right) \geq \liminf _{t \rightarrow \infty} \cos \theta_{j_{0}}(t) \\
& =\cos \left[\frac{\pi}{2}+c(D(\sigma, \tau))+\int_{I(\sigma, \tau)} \kappa d s\right]=\sin L(\sigma, \tau) .
\end{aligned}
$$

Next consider the case where $\beta_{j, t}$ for each $j$ tends to some $\hat{d}_{j}$-ray $\beta_{j}$ at $t \rightarrow \infty$. Since $\theta_{j}(t)$ tends to zero as $t \rightarrow \infty$, which follows from [Co2] (see also Lemma 3.2), we observe by setting $E_{j}:=\cup_{t} E_{j, t}$ that

$$
\begin{aligned}
c(D(\sigma, \tau)) & =\lim _{j \rightarrow \infty} c\left(E_{j}\right)=\lim _{j \rightarrow \infty} \lim _{t \rightarrow \infty} c\left(E_{j, t}\right)=\lim _{j \rightarrow \infty} \lim _{t \rightarrow \infty}\left[2 \pi-\kappa\left(E_{j, t}\right)\right] \\
& =\lim _{j \rightarrow \infty}\left[\pi-\kappa\left(E_{j}\right)\right] .
\end{aligned}
$$

We denote by $\kappa_{j}$ the sum of integrals of geodesic curvature of $\beta_{j}$ and of exterior angles at vertices of $\beta_{j}$ relative to $E_{j}$ and denote by $\psi_{j}$ the inner angle of $E_{j}$ at $\lim _{t \rightarrow \infty} \tau(s(t))$. Then by the definition of $\kappa(\cdot)$,

Hence

$$
\kappa\left(E_{i}\right)=2 \pi-\psi_{j}+\kappa_{j}+\int_{I(\sigma, \tau)} \kappa d s .
$$

$$
L(\sigma, \tau)=-c(D(\sigma, \tau))-\int_{I(\sigma, \tau)} \kappa d s=\lim _{j \rightarrow \infty}\left(\pi-\psi_{j}+\kappa_{j}\right) .
$$

Since $\kappa_{j} \geq 0$ and $\psi_{\jmath} \leq \pi / 2$ for each $j$, we have $L(\sigma, \tau) \geq \pi / 2$ and hence $L(\sigma, \tau)=\pi / 2$. There exists a monotone and divergent sequence $\left\{t_{j}\right\}$ such that

$$
\liminf _{t \rightarrow \infty} \frac{\hat{d}\left(\sigma(t), m_{t}\right)}{t}=\lim _{j \rightarrow \infty} \frac{\hat{d}\left(\sigma\left(t_{j}\right), m_{t j}\right)}{t_{j}}, \quad \lim _{\jmath \rightarrow \infty} \frac{L\left(\beta_{j, t_{j}}\right)}{t_{j}}=1 \quad \text { and } \quad \beta_{j, t_{j}} \subset D_{t_{j}}
$$


because $\beta_{j, t}$ tends to $\beta_{j}$ as $t \rightarrow \infty$ and $\cup D_{t}=D(\sigma, \tau)$. Since $\hat{d}\left(\sigma\left(t_{j}\right), m_{t_{j}}\right) \geq$ $L\left(\beta_{j, t_{j}}\right)$, we have

$$
\liminf _{t \rightarrow \infty} \frac{\hat{d}\left(\sigma(t), m_{t}\right)}{t} \geq 1
$$

Thus in either case

$$
\liminf _{t \rightarrow \infty} \frac{\hat{d}\left(\sigma(t), m_{t}\right)}{t} \geq \sin L(\sigma, \tau)=\sin \frac{L(\sigma, \gamma)}{2} .
$$

In the same way we have

$$
\liminf _{t \rightarrow \infty} \frac{\hat{d}\left(m_{t}, \gamma(t)\right)}{t} \geq \sin \frac{L(\sigma, \gamma)}{2} .
$$

These formulas complete the proof.

Remark 2.3. In the proof of Lemma 2.1 if $\theta(t), \varphi(t)$ are the inner angles of $D_{t}$ at $\sigma(t), \gamma(t)$, then Lemma 2.2 and the formula ( $\left.\sharp\right)$ in the proof of Lemma 2.1 imply

$$
\lim _{t \rightarrow \infty} \theta(t)=\lim _{t \rightarrow \infty} \varphi(t)=\frac{\pi-\min \{L(\sigma, \gamma), \pi\}}{2} .
$$

Lemmas 2.1 and 2.2 imply the following

Proposition 2.4. For any rays $\sigma$ and $\gamma$ with $n(\sigma)=n(\gamma)$, we have

$$
\lim _{t \rightarrow \infty} \frac{\hat{d}(\sigma(t), \gamma(t))}{t}=2 \sin \frac{\min \{L(\sigma, \gamma), \pi\}}{2} .
$$

Proof of Theorem A1. For an arbitrary given monotone and divergent sequence $\left\{t_{j}\right\}$ of positive numbers, let $\alpha_{j}$ be a minimizing segment of $M$ from $\sigma\left(t_{j}\right)$ to $\gamma\left(t_{j}\right)$. If there exists a subsequence $\left\{\alpha_{k}\right\}$ of $\left\{\alpha_{j}\right\}$ such that $\alpha_{k}$ tends to a straight line $\alpha$, then the triangle inequality implies that

$$
\lim _{k \rightarrow \infty} \frac{d\left(\sigma\left(t_{k}\right), \gamma\left(t_{k}\right)\right)}{t_{k}}=2
$$

and moreover $d_{\infty}(\sigma(\infty), \gamma(\infty)) \geq \pi$ by Proposition 1.3 and Theorem 1.4.

We consider the case where there exists a subsequence $\left\{\alpha_{k}\right\}$ of $\left\{\alpha_{j}\right\}$ such that each subsequence of $\left\{\alpha_{k}\right\}$ diverges. Then it follows that $n(\sigma)$ $=n(\gamma)$. Take a tube $U \in \mathscr{U}_{\sigma, \gamma}\left(e_{n(\sigma)}\right)$. For each sufficiently large $k, \alpha_{k}$ is contained in one of the domains $D(\sigma, \gamma)$ and $D(\gamma, \sigma)$. Without loss of 
generality we may assume that each $\alpha_{k}$ is contained in $D(\sigma, \gamma)$. Since $d\left(\sigma\left(t_{k}\right), \gamma\left(t_{k}\right)\right)=L\left(\alpha_{k}\right)=\hat{d}\left(\sigma\left(t_{k}\right), \gamma\left(t_{k}\right)\right)$, we have

$$
\lim _{k \rightarrow \infty} \frac{d\left(\sigma\left(t_{k}\right), \gamma\left(t_{k}\right)\right)}{t_{k}}=2 \sin \frac{\min \{L(\sigma, \gamma), \pi\}}{2}
$$

by Proposition 2.4. On the other hand if $\hat{d}^{\prime}$ denotes the inner distance of $D(\gamma, \sigma)$, then since $d\left(\sigma\left(t_{k}\right), \gamma\left(t_{k}\right)\right) \leq \hat{d}^{\prime}\left(\sigma\left(t_{k}\right), \gamma\left(t_{k}\right)\right)$, we have

$$
\lim _{k \rightarrow \infty} \frac{d\left(\sigma\left(t_{k}\right), r\left(t_{k}\right)\right)}{t_{k}} \leq \lim _{k \rightarrow \infty} \frac{\hat{d}^{\prime}\left(\sigma\left(t_{k}\right), \gamma\left(t_{k}\right)\right)}{t_{k}}=2 \sin \frac{\min \{L(\gamma, \sigma), \pi\}}{2} .
$$

Therefore $\min \{L(\sigma, \gamma), \pi\} \leq \min \{L(\gamma, \sigma), \pi\}$ and

$$
\lim _{k \rightarrow \infty} \frac{d\left(\sigma\left(t_{k}\right), \gamma\left(t_{k}\right)\right)}{t_{k}}=2 \sin \frac{\min \left\{d_{\infty}(\sigma(\infty), \gamma(\infty)), \pi\right\}}{2} .
$$

By the arbitrariness of $\left\{t_{j}\right\}$ this completes the proof.

Proof of Theorem A2. There are sequences $\left\{\sigma_{i}\right\}$ and $\left\{\gamma_{i}\right\}$ of rays from $c$ such that $d_{\infty}\left(\sigma_{i}(\infty), \gamma_{i}(\infty)\right)$ tends to Diam $M(\infty)$. Moreover by Theorem A1

$$
\liminf _{t \rightarrow \infty} \frac{\operatorname{Diam} S(t)}{t} \geq \lim _{t \rightarrow \infty} \frac{d\left(\sigma_{i}(t), \gamma_{i}(t)\right)}{t}=2 \sin \frac{\min \left\{d_{\infty}\left(\sigma_{i}(\infty), \gamma_{i}(\infty)\right), \pi\right\}}{2}
$$

Therefore

$$
\liminf _{t \leftarrow \infty} \frac{\operatorname{Diam} S(t)}{t} \geq 2 \sin \frac{\min \{\operatorname{Diam} M(\infty), \pi\}}{2}
$$

If Diam $M(\infty)=\infty$, then the triangle inequality implies

$$
\limsup _{t \rightarrow \infty} \frac{\operatorname{Diam} S(t)}{t} \leq 2=2 \sin \frac{\min \{\operatorname{Diam} M(\infty), \pi\}}{2} .
$$

Next we consider the case where Diam $M(\infty)<\infty$. Then $M$ has exactly one end. The triangle inequality implies that

$$
\operatorname{Diam} S(t)-\operatorname{Diam} S\left(t^{\prime}\right) \leq 2\left(t-t^{\prime}\right)
$$

for all $t \geq t^{\prime} \geq 0$. Moreover the set of nonexceptional $t$-values is dense in $[0, \infty)$. Hence there is a monotone and divergent sequence $\left\{t_{i}\right\}$ of nonexceptional $t$-values such that

$$
\limsup _{t \rightarrow \infty} \frac{\operatorname{Diam} S(t)}{t}=\lim _{i \rightarrow \infty} \frac{\operatorname{Diam} S\left(t_{i}\right)}{t_{i}} .
$$


If Diam $M(\infty)=0$, then by Lemma 1.7

$$
\lim _{i \rightarrow \infty} \frac{\operatorname{Diam} S\left(t_{i}\right)}{t_{i}} \leq \lim _{i \rightarrow \infty} \frac{L\left(S\left(t_{i}\right)\right)}{t_{i}}=2 \pi \chi(M)-c(M)=0 .
$$

Accordingly we assume that Diam $M(\infty)>0$. We get a pair of two points $p_{i}$ and $q_{i}$ in $S\left(t_{i}\right)$ such that $d\left(p_{i}, q_{i}\right)=$ Diam $S\left(t_{i}\right)$, and minimizing segments $\sigma_{i}, \gamma_{i}:\left[0, t_{i}\right] \rightarrow M$ from points in $c$ to $p_{i}, q_{i}$ such that $d\left(\sigma_{i}(t), c\right)=d\left(\gamma_{i}(t), c\right)$ $=t$ for all $t \in\left[0, t_{i}\right]$. There is a subsequence $\left\{t_{j}\right\}$ of $\left\{t_{i}\right\}$ such that $\sigma_{j}, \gamma_{j}$ tend to some rays $\sigma, \gamma$. The triangle inequality implies that

$$
d\left(p_{j}, q_{j}\right) \leq d\left(\sigma\left(t_{j}\right), \gamma\left(t_{j}\right)\right)+d\left(p_{j}, \sigma\left(t_{j}\right)\right)+d\left(q_{j},\left(t_{j}\right)\right)
$$

and then we have

$$
\begin{aligned}
\limsup _{t \rightarrow \infty} & \frac{\operatorname{Diam} S(t)}{t}=\lim _{j \rightarrow \infty} \frac{\operatorname{Diam} S\left(t_{j}\right)}{t_{j}}=\lim _{j \rightarrow \infty} \frac{d\left(p_{j}, q_{j}\right)}{t_{j}} \\
\leq & \lim _{j \rightarrow \infty} \frac{d\left(\sigma\left(t_{j}\right), \gamma\left(t_{j}\right)\right)}{t_{j}}+\limsup _{j \rightarrow \infty} \frac{d\left(p_{j}, \sigma\left(t_{j}\right)\right)}{t_{j}}+\limsup _{j \rightarrow \infty} \frac{d\left(q_{j}, \gamma\left(t_{j}\right)\right)}{t_{j}} .
\end{aligned}
$$

On the other hand, the assumption $0<$ Diam $M(\infty)<\infty$ implies that $M(\infty)$ is isometric to a circle. Hence for any small $\varepsilon>0$ there are four different rays $\sigma^{-}, \sigma^{+}, \gamma^{-}$and $\gamma^{+}$from $c$ such that $\sigma \subset D\left(\sigma^{-}, \sigma^{+}\right), \gamma \subset D\left(\gamma^{-}, \gamma^{+}\right)$, $L\left(\sigma^{-}, \sigma^{+}\right)<\varepsilon / 2$ and $L\left(\gamma^{-}, \gamma^{+}\right)<\varepsilon / 2$. Then for all sufficiently large $j, p_{j} \in$ $D\left(\sigma^{-}, \sigma^{+}\right)$and $q_{j} \in D\left(\gamma^{-}, \gamma^{+}\right)$and hence

$$
d\left(p_{j}, \sigma\left(t_{j}\right)\right) \leq L\left(S\left(t_{j}\right) \cap D\left(\sigma^{-}, \sigma^{+}\right)\right) \text {and } d\left(q_{j}, \gamma\left(t_{j}\right)\right) \leq L\left(S\left(t_{j}\right) \cap D\left(\gamma^{-}, \gamma^{+}\right)\right) .
$$

Therefore, by Theorem A1 and Lemma 1.7

$$
\begin{aligned}
\limsup _{t \rightarrow \infty} \frac{\operatorname{Diam} S(t)}{t} & \leq 2 \sin \frac{\min \{d(\sigma(\infty), \gamma(\infty)), \pi\}}{2}+L\left(\sigma^{-}, \sigma^{+}\right)+L\left(\gamma^{-}, \gamma^{+}\right) \\
& \leq 2 \sin \frac{\min \{\operatorname{Diam} M(\infty), \pi\}}{2}+\varepsilon .
\end{aligned}
$$

By the arbitrariness of $\varepsilon>0$, this completes the proof.

\section{§3. Asymptotic behavior of the angles}

First we state a few lemmas used in the proof of Theorems B1, C1, and C2. The following lemma is obvious by the Gauss-Bonnet theorem.

LEMMA 3.1. Let $\sigma$ and $\gamma$ be rays with $n(\sigma)=n(\gamma)=i$ and $D$ a domain in $M$ bounded by piecewise smooth curves $c_{1}, \cdots, c_{m}(m \geq 1)$ such that 
$c_{1}(-a-t)=\sigma\left(t_{0}+t\right)$ and $c_{1}(a+t)=\gamma\left(t_{1}+t\right)$ hold for all $t \geq 0$ and for some constants $a, t_{0}, t_{1} \geq 0$ and $c_{2}, \cdots, c_{m}$ are simple closed (see Figure 3.1.f). Then we have

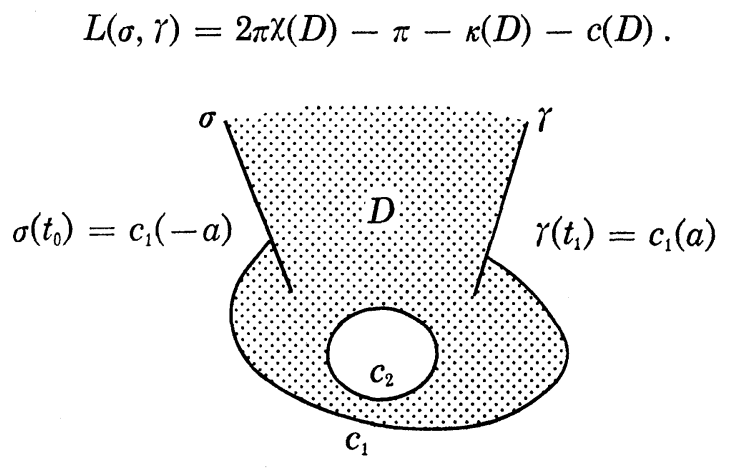

Figure 3.1.f

We define the tangent cone by

$$
C_{p}(v, \theta):=\left\{u \in T_{p} M-\{0\} ; \Varangle(u, v)<\theta\right\}
$$

for $v \in T_{p} M-\{0\}, 0<\theta<\pi / 2$. For a compact subset $K$ of $M$ and for a point $p$ in $M$, we set

$W_{p}(K):=\left\{\dot{\sigma}(0) \in S_{p} M ; \sigma\right.$ is a minimizing segment from $p$ to a point in $\left.K\right\}$, where $S_{p} M$ denotes the set of all unit vectors in $T_{p} M$.

The following lemma is a modification of Lemma 1.2 in [SST].

LEMma 3.2 (1.2, [SST]). Let $K$ be an arbitrary given compact subset of $M$ and $\varepsilon>0$ be an arbitrary small number. There exists a radius $R(K, \varepsilon)$ $>0$ such that for any $p \in M$ with $d(p, K)>R(K, \varepsilon)$, we can choose $v_{p} \in S_{p} M$ satisfying

$$
W_{p}(K) \subset C_{p}\left(v_{p}, \varepsilon\right) .
$$

The following lemma is due to Cohn-Vossen [Co1].

Lemma 3.3 ([Co1]). Assume that $s_{i}(M)>0$ for some $i$. For any compact subset $L$ of $M$ there exists a tube $U \in \mathscr{U}\left(e_{i}\right)$ such that $M-U$ contains $K$ and is convex.

Note that if $s_{i}(M)>0$, then any tube in $\mathscr{U}\left(e_{i}\right)$ is expanding in the sense of Busemann (section 43, [Bu]), which shows Lemma 3.3.

Let $\left\{p_{j}\right\}$ be an arbitrary given sequence of points in $M$ such that 
$\varphi\left(p_{j}\right)$ tends to a fixed end $e_{j}$, where $\varphi: M \rightarrow N-\left\{e_{1}, \cdots, e_{k}\right\}$ is the homeomorphism as above. Let $\rho$ and $\rho^{\prime}$ be constants such that $0 \leq \rho<+\infty$, $0 \leq \rho^{\prime} \leq+\infty, \rho \leq \rho^{\prime}$ and $\rho+\rho^{\prime}=s_{i}(M)$. For each $j$ we get arbitrary different rays $\sigma_{j}$ and $\gamma_{j}$ emanating from $p_{j}$ such that $n\left(\sigma_{j}\right)=n\left(\gamma_{j}\right)=i$,

$$
\rho=L\left(\sigma_{j}, \gamma_{j}\right) \text { and } \rho^{\prime}=L\left(\gamma_{j}, \sigma_{j}\right) .
$$

Note that all $\sigma_{j}(\infty)$ (resp. $\gamma_{j}(\infty)$ ) are not necessarily same.

We will investigate the asymptotic behavior of the angles $\Varangle\left(\dot{\sigma}_{j}(0), \dot{\gamma}_{j}(0)\right)$ and prove Theorem B1 (resp. C1 and C2) under the condition $\rho=d_{\infty}(x, y)$ $<\pi$ (resp. $\rho=0$ ). Choosing a subsequence of $\left\{p_{j}\right\}$, one of the following cases occurs (we write the subsequence the same notation $\left\{p_{j}\right\}$ ).

Case 1: All subsequences of $\left\{\sigma_{j}\right\},\left\{\gamma_{j}\right\}$ diverge. In this case, there exists for a fixed tube $U \in \mathscr{U}\left(e_{i}\right)$ a number $j_{0}$ such that $\sigma_{j} \cup \gamma_{j}$ for each $\geq j_{0}$ is contained in $U$ and bounds domains of $U$. By Lemma 3.1 we can choose one of these domains, $D_{j}$, such that

$$
\rho=2 \pi \chi\left(D_{j}\right)-\pi-\kappa\left(D_{j}\right)-c\left(D_{j}\right) .
$$

Case 2: Each subsequence of $\left\{\sigma_{j}\right\}$ diverges and $\left\{\gamma_{j}\right\}$ converges to some straight line $r$. The existence of the straight line implies that $s_{i}(M) \geq 2 \pi$ by Proposition 1.3 and hence the assumption of Lemma 3.3 is satisfied. We get a tube $U \in \mathscr{U}\left(e_{i}\right)$ such that $M-U$ is convex and each $\gamma_{j}$ intersects $M-U$. There exists a number $j_{0}$ such that $\sigma_{j}$ is contained in $U$ for all $j \geq j_{0}$. We get an open half plane $D_{j}$ for $j \geq j_{0}$ in $U$ which is a connected component of $U-\left(\sigma_{j}([0, \infty)) \cup \gamma_{j}([0, \infty))\right)$ such that the equality $(*)$ holds.

Case 3: $\left\{\sigma_{j}\right\}$ and $\left\{\gamma_{j}\right\}$ converge to some straight lines $\sigma$ and $\gamma$ respectively. In this case, $\Varangle\left(\dot{\sigma}_{j}(0), \dot{\gamma}_{j}(0)\right)$ tends to zero as $j \rightarrow \infty$ by Lemma 3.2.

The following lemma is the key to this and the next section.

LEMma 3.4. In Cases 1 and 2 we denote by $\theta_{j}$ the inner angle of $D_{j}$ at $p_{j}$. Then (1), (2) and (3) hold (see Figure 3.4.f).

(1) In Case 1 if $\partial U \cap \mathrm{Cl}\left(D_{j}\right)=\varnothing$ for all $j$, then

$$
\limsup _{j \rightarrow \infty} \theta_{j} \leq \rho .
$$

(2) In Case 1 if $\partial U \subset \mathrm{Cl}\left(D_{3}\right)$ for all $j$, then

$$
\lim _{j \rightarrow \infty} \theta_{j}=2 \pi-\rho^{\prime} \text {. }
$$

(3) In Case 2, 


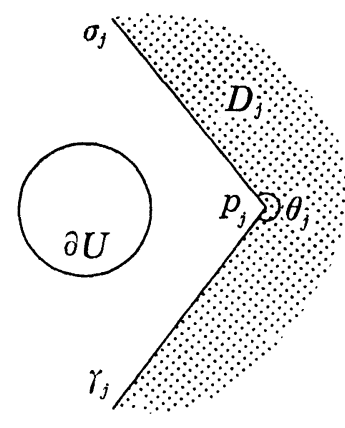

Case 1: $\partial U \cap \mathrm{Cl}\left(D_{j}\right)=\emptyset$

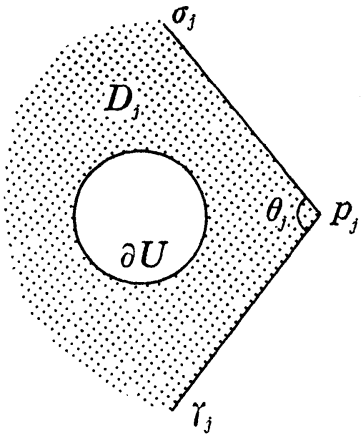

Case 1: $\partial U \subset \mathrm{Cl}\left(D_{j}\right)$

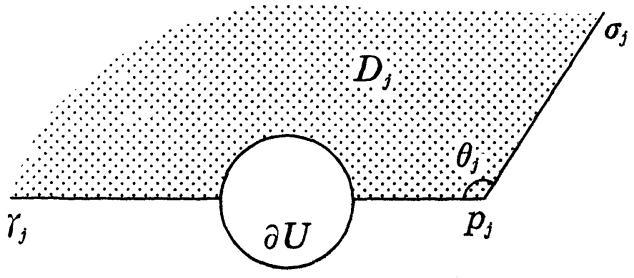

Case 2

Figure 3.4.f

$$
\limsup _{j \rightarrow \infty} \theta_{j} \leq \rho .
$$

Proof of (1). By $(*)$ we have

$$
\rho=\theta_{j}-c\left(D_{j}\right)
$$

for all sufficiently large $j$. For any positive $\varepsilon$ there exists a compact subset $K$ of $U$ such that

$$
\int_{U-K} G^{+} d M<\varepsilon,
$$

where $G^{+}(x):=\max \{G(x), 0\}$. Since $D_{j}$ does not intersect $K$ for all suffciently large $j$, we have

$$
c\left(D_{j}\right)<\varepsilon \text { and hence } \theta_{j}<\rho+\varepsilon
$$

for all sufficiently large $j$. This completes the proof of (1).

Proof of (2). For all sufficiently large $j(*)$ implies that

$$
\rho=\theta_{j}-2 \pi-\kappa(U)-c\left(D_{j}\right) .
$$

Moreover $c\left(D_{j}\right)$ tends to $c(U)$ by $\cup D_{j}=U$. Hence 


$$
\lim _{j \rightarrow \infty} \theta_{j}=\rho+2 \pi+\kappa(U)+c(U)=2 \pi-\rho^{\prime},
$$

because $\rho+\rho^{\prime}=s_{i}(M)$. This completes the proof of (2).

Proof of (3). It suffices to show that there exists a subsequence $\left\{\theta_{k}\right\}$ of $\left\{\theta_{j}\right\}$ such that

$$
\limsup _{k \rightarrow \infty} \theta_{k} \leq \rho .
$$

For a geodesic $\alpha$ passing through $M-U$, we set

$$
\begin{aligned}
& \xi(\alpha):=\alpha(\inf \{t ; \alpha(t) \in M-U\}), \\
& \eta(\alpha):=\alpha(\sup \{t ; \alpha(t) \in M-U\}) .
\end{aligned}
$$

By the convexity of $M-U, \xi\left(\gamma_{j}\right)$ and $\eta\left(\gamma_{j}\right)$ tend to $\xi(\gamma)$ and $\eta(\gamma)$ respectively. The arc $I_{j}:=\mathrm{Cl}\left(D_{j}\right) \cap \partial U$ is one of the two subarcs of $\partial U$ joining $\xi\left(\gamma_{j}\right)$ and $\eta\left(\gamma_{j}\right)$. There is a subsequence $\left\{I_{k}\right\}$ of $\left\{I_{j}\right\}$ converging to a subarc $I$ of $\partial U$, which joins $\xi(\gamma)$ and $\eta(\gamma)$. We get an open half plane $H$ in $U$ which is a connected component of $U-\gamma((-\infty, \infty))$ such that $\mathrm{Cl}(H) \cap \partial U$ $=I$. By (*), we have

$$
c\left(D_{k}\right)=\pi-\kappa\left(D_{k}\right)-\rho .
$$

For an arbitrary positive $\varepsilon$, we get a compact subset $K$ of $U$ such that

$$
\int_{U-K} G^{+} d M<\varepsilon
$$

Then

$$
\pi-\kappa\left(D_{k}\right)-\rho=c\left(D_{k}\right)<c\left(D_{k} \cap K\right)+\varepsilon<c(H \cap K)+2 \varepsilon
$$

for all sufficiently large $k$. This means that $c(H)$ is a finite value. Thereby we may assume that $c(H \cap K)<c(H)+\varepsilon$ (we replace $K$ by a larger compact set if necessary). Since $\gamma$ is a straight line, we have $c(H) \leq-\kappa(H)$ by Lemmas 1.2 and 3.1. Hence

$$
\pi-\kappa\left(D_{k}\right)-\rho<-\kappa(H)+3 \varepsilon
$$

for all sufficiently large $k$. On the other hand, it follows from the definition of $\kappa(\cdot)$ that $\kappa\left(D_{k}\right)-\left(\pi-\theta_{k}\right)$ tends to $\kappa(H)$ and hence

$$
\kappa\left(D_{k}\right)-\pi+\theta_{k}-\kappa(H)<\varepsilon
$$

for all sufficiently large $k$. Therefore

$$
\theta_{k}<\rho+4 \varepsilon
$$


for all sufficiently large $k$. This completes the proof of (3).

Proof of Theorem B1. Now, if $d_{\infty}(x, y) \geq \pi$, then the inequality of Theorem B1 is obvious. Accordingly, we assume that $d_{\infty}(x, y)<\pi$, and set $i:=n(x)=n(y)$. If there exists a subsequence $\left\{p_{k}\right\}$ of $\left\{p_{j}\right\}$ such that $\varphi\left(p_{k}\right)$ tends to an end different from $e_{i}$ as $k \rightarrow \infty$, then $\sigma_{k}$ and $\gamma_{k}$ intersect $\partial U$ for all sufficiently large $k$ and for a fixed tube $U \in \mathscr{U}\left(e_{i}\right)$. Lemma 3.2 implies

$$
\lim _{k \rightarrow \infty} \Varangle\left(\dot{\sigma}_{k}(0), \dot{\gamma}_{k}(0)\right)=0 .
$$

We consider the case where $\varphi\left(p_{j}\right)$ tends to $e_{i}$. Set $\rho:=d_{\infty}(x, y)$ and $\rho^{\prime}:=s_{i}(M)-\rho$. Then the assumptions $d_{\infty}(x, y)<\pi$ and $s_{i}(M) \geq 2 \pi$ imply $0 \leq \rho<\pi<\rho^{\prime} \leq+\infty$. If there exists a subsequence $\left\{p_{k}\right\}$ of $\left\{p_{j}\right\}$ such that the assumption of Lemma 3.4 (1) or (3) is satisfied for $\left\{p_{k}\right\}$, then since $\Varangle\left(\dot{\sigma}_{k}(0), \dot{\gamma}_{k}(0)\right) \leq \theta_{k}$ for all $k$, we have

$$
\limsup _{k \rightarrow \infty} \Varangle\left(\dot{\sigma}_{k}(0), \dot{\gamma}_{k}(0)\right) \leq d_{\infty}(x, y) .
$$

If there exists a subsequence $\left\{p_{k}\right\}$ of $\left\{p_{j}\right\}$ such that the assumption of Lemma 3.4 (2) is satisfied for $\left\{p_{k}\right\}$, then (**) holds because $2 \pi-\rho^{\prime}=2 \pi-$ $s_{i}(M)+\rho \leq \rho=d_{\infty}(x, y)$. If Case 3 occurs for some subsequence $\left\{p_{k}\right\}$ of $\left\{p_{j}\right\}$, then $\Varangle\left(\dot{\sigma}_{k}(0), \dot{\gamma}_{k}(0)\right)$ tends to zero. By the arbitrariness of $\left\{p_{j}\right\}$ this completes the proof.

Proof of Theorem B2. If there is a monotone and divergent sequence $\left\{t_{j}\right\}$ of positive numbers such that $\gamma_{t j}$ tends to some straight line $\gamma_{\infty}$, then $\left.\Varangle\left(\dot{\sigma}_{j}\right), \dot{\gamma}_{t}(0)\right)$ tends to $\pi$ as $j \rightarrow \infty$ by Lemma 3.2 and moreover $d_{\infty}(\sigma(\infty), \gamma(\infty))$ $\geq \pi$ by Proposition 1.3 and Theorem 1.4. The proof is completed in this case.

Next we consider the case where $\left\{\gamma_{t_{j}}\right\}$ diverges for any monotone and divergent sequence $\left\{t_{j}\right\}$. We get a tube $U \in \mathscr{U}_{\sigma, r}\left(e_{i}\right)$ and a monotone and divergent sequence $\left\{t_{j}\right\}$ such that each $\gamma_{t_{j}}$ is contained in $U$. Without loss of generality we may assume that each $\gamma_{t_{j}}$ is contained in $D(\sigma, \gamma)$. It follows from Lemma 1.9 that

$$
\lim _{j \rightarrow \infty} \chi\left(\dot{\sigma}(t), \dot{\gamma}_{t}(0)\right)=\min \{L(\sigma, \gamma), \pi\} .
$$

It suffices to show that $\min \{L(\sigma, \gamma), \pi\}=\min \left\{d_{\infty}(\sigma(\infty), \gamma(\infty)), \pi\right\}$. Since each $\gamma_{t_{j}}$ is contained in $D(\sigma, \gamma)$, there is a monotone and divergent sequence $\left\{s_{k}(j)\right\}_{k}$ depending on $j$ such that for any $k$ some minimizing segment from 
$\gamma_{t j}(\varepsilon)$ to $\gamma\left(s_{k}(j)\right)$ is contained in $D(\sigma, \gamma)$ and hence $\hat{d}\left(\gamma_{t j}(\varepsilon), \gamma\left(s_{k}(j)\right)\right)=d\left(\gamma_{t}(\varepsilon)\right.$, $\left.\gamma\left(s_{k}(j)\right)\right)$, where $\varepsilon$ is any fixed positive number. This implies that $\hat{F}_{r} \circ \sigma\left(t_{j}\right)$ $=F_{\gamma} \circ \sigma\left(t_{j}\right)$ for all $j$. Thus from Lemma 1.10 and Theorem 1.11

$$
\begin{aligned}
\cos \min \{L(\sigma, \gamma), \pi\} & =\lim _{j \rightarrow \infty} \frac{\hat{F}_{\gamma} \circ \sigma\left(t_{j}\right)}{t_{j}}=\lim _{j \rightarrow \infty} \frac{F_{\gamma} \circ \sigma\left(t_{j}\right)}{t_{j}} \\
& =\cos \min \left\{d_{\infty}(\sigma(\infty), \gamma(\infty)), \pi\right\} .
\end{aligned}
$$

This completes the proof.

REMARK 3.5. If $s_{i}(M)<2 \pi$ for some $i$, then the inequality of Theorem B1 does not necessarily hold.

Indeed we consider a surface $M$ with $0<s_{i}(M)<2 \pi$ which contains a flat tube $U \in \mathscr{U}\left(e_{i}\right)$. Since the tube $U$ can be embedded in the Euclidean 3 -space, we can choose a pair of rays $\alpha$ and $\beta$ in $U$ such that for any $s, t \geq 0$ there are exactly two minimizing segment from $\alpha(s)$ to $\beta(t)$ contained in $U$. For any $s \geq 0$ there are two different rays $\sigma_{s}$ and $\gamma_{s}$ emanating from $\alpha(s)$ which are asymptotic to $\beta$ (see Figure 3.5.f).

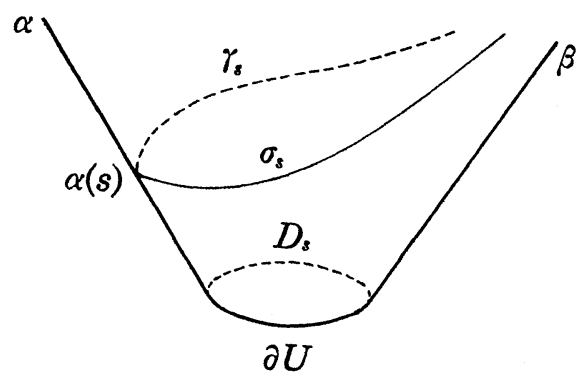

Figure 3.5.f

We have $\sigma_{s}(\infty)=\gamma_{s}(\infty)$ for each $s \geq 0$ by Theorem 1.4. Let $D_{s}$ for $s \geq 0$ be a domain of $U$ bounded by $\sigma_{s} \cup \gamma_{s}$ such that $D_{s}$ contains $\beta$, then $\left\{D_{s}\right\}$ is a monotone increasing sequence with $U D_{s}=U$. From Lemma 3.1, if $\theta_{s}$ denotes the inner angle of $D_{s}$ at $\alpha(s)$, then for each $s \geq 0$

$$
0=2 \pi \chi\left(D_{s}\right)-\pi-\kappa(D)-c(D)=-2 \pi-\kappa(U)+\theta_{s}
$$

and hence

$$
\theta_{s}=2 \pi-s_{i}(M)
$$

because $c(U)=0$. Since $0<s_{i}(M)<2 \pi$, 


$$
\Varangle\left(\dot{\sigma}_{s}(0), \dot{\gamma}_{s}(0)\right)=\min \left\{s_{i}(M), 2 \pi-s_{i}(M)\right\}>0
$$

for all $s \geq 0$, which contradicts the inequality of Theorem B1.

\section{§4. Critical points of Busemann functions}

In Lemma 3.4 we assume that $\rho=0$ and $\rho^{\prime}=s_{i}(M)$. Then we have the following directly.

LemMA 4.1. Let $\left\{p_{j}\right\}$ be an arbitrary sequence of points in $M$ such that $\varphi\left(p_{j}\right)$ tends to an end $e_{i}$ as $j \rightarrow \infty$ and let $\sigma_{j}$ and $\gamma_{j}$ be rays emanating from $p_{j}$ such that $\sigma_{j}(\infty)=\gamma_{j}(\infty) \in M_{i}(\infty)$ for each $j$. Then there exists a subsequence $\left\{p_{k}\right\}$ of $\left\{p_{j}\right\}$ such that (1) or (2) holds.

(1) $\Varangle\left(\dot{\sigma}_{k}(0), \dot{\gamma}_{k}(0)\right)$ tends to zero as $k \rightarrow \infty$.

(2) The sequence $\left\{p_{k}\right\}$ satisfies the assumption of Lemma 3.4 (2) and $\theta_{k}$ tends to $2 \pi-s_{i}(M)$.

Note that $s_{i}(M) \leq 2 \pi$ holds whenever (2) occurs.

Proof of Theorem C1. Suppose that $\operatorname{Crit}(M)$ is unbounded. Then there is a sequence $\left\{p_{j}\right\}$ of points in $\operatorname{Crit}(M)$ such that $\varphi\left(p_{j}\right)$ tends to some end $e_{i}$. Let $\alpha_{j}$ be a ray such that $p_{j}$ is a critical point of the Busemann function $F_{a j}$. For each $j$ we get a ray $\sigma_{j}$ emanating from $p_{j}$ asymptotic to $\alpha_{j}$.

Now, suppose that $s_{i}(M)=0$ or $s_{i}(M) \geq 2 \pi$. We get an arbitrary sequence $\left\{\gamma_{j}\right\}$ of rays such that each $\gamma_{j}$ emanates from $p_{j}$ and is asymptotic to $\alpha_{j}$. Then Lemma 4.1 implies that some subsequence of $\left\{\Varangle\left(\dot{\sigma}_{j}(0), \dot{\gamma}_{j}(0)\right)\right\}$ converges to zero as $j \rightarrow \infty$. This contradicts that every $p_{j}$ is a critical point.

Thus we consider the case where $0<s_{i}(M)<2 \pi$. Set

$$
\theta:=\min \left\{s_{i}(M), 2 \pi-s_{i}(M)\right\} .
$$

It follows that $0<\theta<\pi$. We get three different vectors $v_{j}^{a} \in S_{p j} M$ for $a=0,1,2$ such that $v_{j}^{0}:=\dot{\sigma}_{j}(0)$ and $\Varangle\left(v_{j}^{0}, v_{j}^{1}\right)=\Varangle\left(v_{j}^{0}, v_{j}^{2}\right)=\theta$. Applying Lemma 4.1 to $\sigma_{j}$ and every ray from $p_{j}$ asymptotic to $\alpha_{j}$, we obtain that for any small $\varepsilon>0$ there is a number $j(\varepsilon)$ such that

$$
V_{p_{j}}\left(F_{\alpha_{j}}\right) \subset \bigcup_{a=0,1,2} C_{p_{j}}\left(v_{j}^{a}, \varepsilon\right)
$$

for each $j \geq j(\varepsilon)$, where $V_{p}(f)$ is as in section 0 . Since $p_{j}$ is a critical point of $F_{\alpha_{j}}$, the sets $V_{p_{j}}\left(F_{\alpha_{j}}\right) \cap C_{p_{j}}\left(v_{j}^{1}, \varepsilon\right)$ and $V_{p_{j}}\left(F_{\alpha_{j}}\right) \cap C_{p_{j}}\left(v_{j}^{2}, \varepsilon\right)$ are nonempty 
for each $j \geq j(\varepsilon)$ and we obtain $\theta \geq \pi / 2$ by the arbitrariness of $\varepsilon>0$. Fix a small $\varepsilon>0$. We get two rays $\tau_{j}$ and $\gamma_{j}$ for $j \geq j(\varepsilon)$ such that

$$
\dot{\tau}_{j}(0) \in V_{p_{j}}\left(F_{a_{j}}\right) \cap C_{p_{j}}\left(v_{j}^{1}, \varepsilon\right) \text { and } \dot{\gamma}_{j}(0) \in V_{p_{j}}\left(F_{\alpha_{j}}\right) \cap C_{p_{j}}\left(v_{j}^{2}, \varepsilon\right) \text {. }
$$

Here $\left\{\sigma_{j}\right\}$ and $\left\{\gamma_{j}\right\}$ (resp. $\left\{\sigma_{j}\right\}$ and $\left\{\tau_{j}\right\}$ ) are satisfy the assumption of Lemma 3.4 (2), hence all subsequences of these diverge. Therefore, for a fixed tube $U \in \mathscr{U}\left(e_{i}\right)$ there is a number $j_{0}$ such that $\sigma_{j}, \tau_{j}$ and $\gamma_{j}$ for every $j \geq j_{0}$ are contained in $U$. For each $j \geq j_{0}$ the set $U-\left(\sigma_{j} \cup \tau_{j} \cup \gamma_{j}\right)$ consists of three connected components. Choose one of these components containing $\partial U$ and denote it by $D_{j}$. Let $E_{j}$ and $F_{j}$ be the closures of the other components. Lemma 4.1 implies that the three inner angles of $D_{j}, D_{j} \cup E_{j}$ and $D_{j} \cup F_{j}$ must tend to $2 \pi-s_{i}(M)$ respectively, which is a contradiction. This completes the proof of Theorem C1.

Proof of Theorem C2. Suppose that $s_{i}(M)=\pi$ for some $i$ and Crit $(M)$ is unbounded. Then there is a divergent sequence $\left\{p_{j}\right\}$ of points in $\operatorname{Crit}(M)$. We may consider the case where $p_{j}$ tends to the end $e_{i}$. Let $\left\{\alpha_{j}\right\}$ be as in the proof of Theorem C1. Take an arbitrary small number $\varepsilon>0$ and vectors $v_{j} \in V_{p_{j}}\left(F_{\alpha_{j}}\right)$ for all $j$. Then by Lemma 4.1 there is a number $j(\varepsilon)$ such that

$$
V_{p_{j}}\left(F_{\alpha_{j}}\right) \subset C_{p_{j}}\left(v_{j}, \varepsilon\right) \cup C_{p_{j}}\left(-v_{j}, \varepsilon\right)
$$

for each $j \geq j(\varepsilon)$. We get arbitrary rays $\sigma_{j}$ and $\gamma_{j}$ for $j \geq j(\varepsilon)$ such that

$$
\dot{\sigma}_{j}(0) \in V_{p_{j}}\left(F_{\alpha_{j}}\right) \cap C_{p_{j}}\left(v_{j}, \varepsilon\right) \text { and } \dot{\gamma}_{j}(0) \in V_{p_{j}}\left(F_{\alpha_{j}}\right) \cap C_{p_{j}}\left(-v_{j}, \varepsilon\right) \text {. }
$$

By Lemma 4.1, $\sigma_{j} \cup \gamma_{j}$ for each sufficiently large $j$ does not intersect $\partial U$ and bounds two domains of $U$ for a fixed tube $U \in \mathscr{U}\left(e_{i}\right)$. Choose one of these domains containing $\partial U$ and denote it by $D_{j}$. Denote the inner angle of $D_{j}$ at $p_{j}$ by $\theta_{j}$. Since $\left\{D_{j}\right\}$ satisfies the assumption of Lemma 3.4 (2), the formula $(*)$ in section 3 holds, that is,

$$
0=\theta_{j}-2 \pi-\kappa(U)-c\left(D_{\jmath}\right) .
$$

By the assumption of Theorem $\mathrm{C} 2$ and by $\cup D_{j}=U$, there is a number $j_{0}$ such that the signs of the Gaussian curvatures at points in $U-D_{j}$ are same for every $j \geq j_{0}$. If the sign is positive, then $c\left(D_{j}\right)<c(U)$ and hence $\theta_{j}<2 \pi+\kappa(U)+c(U)=\pi$ for each $j \geq j_{0}$. If the sign is negative, then $c\left(D_{j}\right)>c(U)$ and hence $\theta_{j}>\pi$ for each $j \geq j_{0}$. Thus, the arbitrariness of $\left\{\sigma_{j}\right\}$ and $\left\{\gamma_{j}\right\}$ yeilds that $V_{p_{j}}\left(F_{\alpha}\right)$ is contained in an open half plane of 
$T_{p} M$ for all sufficiently large $j$, which contradicts that $p_{j}$ is a critical point. This completes the proof of Theorem C2.

Remark 4.2. If $s_{i}(M)=\pi$ for some $i$, then $\operatorname{Crit}(M)$ is not necessarily bounded.

Indeed we consider the surface $M$ as in Remark 3.5 with $s_{i}(M)=\pi$. Let $\alpha, \beta, \sigma_{s}$ and $\gamma_{s}$ be rays in $M$ as in Remark 3.5. Since $\Varangle\left(\sigma_{s}(0), \gamma_{s}(0)\right.$ ) $=\pi, \alpha(s)$ for all $s \geq 0$ are critical points of $F_{\beta}$. This means that $\operatorname{Crit}(M)$ is unbounded. Moreover we observe that $\operatorname{Crit}(M)$ contains a tube in $\mathscr{U}\left(e_{i}\right)$.

\section{REFERENCES}

[BGS] W. Ballmann, M. Gromov and V. Schroeder, Manifolds of Nonpositive Curvature, Progress in Math. 61, Birkhäuser, Boston-Basel Stuttgart, 1985.

[Bu] H. Busemann, The geometry of geodesics, Academic Press, New York, 1955.

[Co1] S. Cohn-Vossen, Kürzeste Wege und Totalkrümmung auf Flächen, Composito Math., 2 (1935), 63-133.

[Co2] - Totalkrümmung und geodätische Linien auf einfach zusammenhängenden offenen volständigen Flächenstücken, Recueil Math. Moscow, 43 (1936), 139 163.

[EO] P. Eberlein and B. O'Neill, Visibility manifolds, Pac. J. Math., 46 (1973), 45110 .

[Fi] F. Fiala, Le problème isopérimètres sur les surface onvretes à courbure positive, Comment. Math. Helv., 13 (1941), 293-346.

[Ha] P. Hartman, Geodesic parallel coordinates in the large, Amer. J. Math., 86 (1964), 705-727.

[Ks] A. Kasue, A compactification of a manifold with asymptotically nonnegative curvature, Ann. scient. Éc. Norm. Sup., 21 (1988), 593-622.

[Md1] M. Maeda, A geometric significance of total curvature on complete open surfaces, Geometry of Geodesics and Related Topics, Advanced Studies in Pure Math., 3 (1984), 451-458, Kinokuniya, Tokyo, 1984.

[Md2] - On the total curvature of noncompact Riemannian manifolds I, Yokohama Math. J., 33 (1985), 93-101.

[Og] T. Oguchi, Total curvature and measure of rays, Proc. Fac. Sci. Tokai Univ., 21 (1986), 1-4.

[Ot] F. Ohtsuka, On a relation between total curvature and Tits metric, Bull. Fac. Sci. Ibaraki Univ., 20 (1988).

[Sg1] K. Shiga, On a relation between the total curvature and the measure of rays, Tsukuba J. Math., 6 (1982), 41-50.

[Sg2] - A relation between the total curvature and the measure of rays, II, Tôhoku Math. J., 36 (1984), 149-157.

[Sh1] K. Shiohama, Busemann function and total curvature, Invent. Math., 53 (1979), 281-297.

[Sh2] - The role of total curvature on complete noncompact Riemannian 2-manifolds, Illinois J. Math., 28 (1984), 597-620.

[Sh3] - Cut locus and parallel circles of a closed curve on a Riemannian plane admitting total curvature, Comment. Math. Helv., 60 (1985), 125-138.

[Sh4] - Total curvatures and minimal areas of complete open surfaces, Proc. 
Amer. Math. Soc., 94 (1985), 310-316.

[Sh5] — An integral formula for the measure of rays on complete open surfaces, J. Differential Geometry, 23 (1986), 197-205.

[SST] K. Shiohama, T. Shioya and M. Tanaka, Mass of rays on complete open surfaces, Pac. J. Math., 143 (1990), 349-358.

[ST] K. Shiohama and M. Tanaka, An isoperimetric problem for infinitely connected complete open surfaces, Geometry of Manifolds, Perspectives in Mathematics, 8 (1989), 317-343, Academic Press.

[Sy1] T. Shioya, On asymptotic behavior of the mass of rays, Proc. Amer. Math. Soc., 108 (1990), 495-505.

[Sy2] - The ideal boundaries of complete open surfaces, to appear in Tôhoku Math. J.

[Sy3] — The ideal boundaries of complete open surfaces admitting total curvature $c(M)=-\infty$, Geometry of Manifolds, Perspectives in Mathematics, 8 (1989) 351364, Academic Press.

Department of Mathematics

Faculty of Science

Fukuoka 812

Japan 